\title{
Measurement of Nightmare Experience, and its Application in Research and Treatment of Psychiatric Disorders
}

Wei Wang* and Jiawei Wang

Department of Clinical Psychology and Psychiatry, School of Medicine, Zhejiang University College of Medicine, Hangzhou, China

\section{Editorial}

Nightmares are typically extended, elaborate dream narratives, and usually occur during rapid eye movement period of sleep. Nightmare experience is accompanied by the disturbed affect culminating in arousal, which usually involves an individual's efforts to avoid threats to survival, security, or physical integrity, but often results in awakening with rapid orientation and alertness [1]. Nightmare experience might also leave the individual negative emotions and cause obvious distress, emotional deregulation, or impairment in social, occupational, or other functions [2].

About $26 \%$ of community adults and $39.8 \%$ of psychiatric patients suffer from weekly nightmares, a frequency that is thought to reflect moderately severe pathology [3]. Many studies have reported a gender difference in nightmare frequencies. For instance, a meta-analysis including 111 independent studies demonstrated that women tended to report nightmares more often than men did but this gender difference was not found in children or in elderly [4]. Further, several candidate variables like dream recall frequency, depression, childhood trauma, and insomnia have been demonstrated to affect the nightmare frequency and to explain this gender difference. In addition, neuroticism trait and gender-specific socialization processes contribute a lot to this gender difference [5].

On the other hand, more detailed, comprehensive description regarding nightmare experience contents are needed besides the nightmare frequency. Keeping this in mind, a group of investigators have developed a Nightmare Experience Questionnaire (NEQ) using both exploratory and confirmatory factor analyses, in 321 Chinese university students who had a life-long nightmare experience [6]. This structure-validated NEQ has provided measures for four factors, namely Physical Effect, Negative Emotion, Meaning Interpretation and Horrible Stimulation. This study has also demonstrated some gender differences on two factors: women scored significantly higher on NEQ Negative Emotion and Meaning Interpretation factors than men did. Interestingly, the nightmare frequency was positively correlated with all the four NEQ factors.

Not surprisingly, nightmares are involved in many psychiatric disorders, indicating that there is a commonly underlying mechanism of psychological dysfunction and nightmare experience. For instance, in a functional magnetic resonance imaging study, scholars [7] used the resting-state paradigm in nightmare disorder patients, and found that the higher values of regional homogeneity in clusters within the left anterior cingulate cortex and right inferior parietal lobule, and lower regional homogeneity values within the left superior and inferior frontal gyri and bilateral middle occipital gyri in these patients. Moreover, NEQ Physical Effect was negatively correlated with regional homogeneity values in anterior cingulate cortex and inferior parietal lobule in these patients.

Before NEQ was developed, the recall of nightmare experience has been used in the treatment of nightmare and related sleep disorders. When treating nightmares, scholars [8] used the imagery rehearsal therapy (IRT) and prazosin, and found that the IRT combined with cognitive-behavioral therapy for insomnia showed more improvement in sleep quality compared to prazosin, or IRT alone. These results also encourage the application of the imagery rehearsal therapy or prazosin as a first-line intervention for post-traumatic stress disorder, or as an adjunctive therapy to reduce suicide risk in patients at risk of suicide with nightmares [9]. It is reasonable that we expect more reliable results using more solid description of nightmare experience in these patients. In this case, the NEQ might provide a great help.

\section{References}

1. American Psychiatric Association (2013) Diagnostic and statistical manual of mental disorders (DSM-5). American Psychiatric Pub.

2. Zadra A, Pilon M, Donderi DC (2006) Variety and intensity of emotions in nightmares and bad dreams. J Nerv Ment Dis 194: 249-254.

3. Levin R, Nielsen TA (2007) Disturbed dreaming, posttraumatic stress disorder and affect distress: A review and neurocognitive model. Psychol Bull 133: 482 528

4. Schredl M, Reinhard I (2011) Gender differences in nightmare frequency: A meta-analysis. Sleep Med Rev 15: 115-121.

5. Schredl M (2014) Explaining the gender difference in nightmare frequency. Am J Psychol 127: 205-213.

6. Chen W, Xu Y, Zhu M, Tang Y, Huang S (2014) Development of a structurevalidated Nightmare Experience Questionnaire in Chinese university students. J Psychiatry 17: 147

7. Shen C, Wang J, Ma G, Zhu Q, He H, et al. (2016) Waking-hour cerebra activations in nightmare disorder: A resting-state functional magnetic resonance imaging study. Psychiatry Clin Neurosci 70: 573-581.

8. Seda G, Sanchez-Ortuno MM, Welsh CH, Halbower AC, Edinger JD (2015) Comparative meta-analysis of prazosin and imagery rehearsal therapy for nightmare frequency, sleep quality, and posttraumatic stress. J Clin Sleep Med 11: 11-22.

9. Nadorff MR, Lambdin KK, Germain A (2014) Pharmacological and nonpharmacological treatments for nightmare disorder. Int Rev Psychiatry 26: 225236 .

*Corresponding author: Wei Wang, Department of Clinical Psychology and Psychiatry, School of Medicine, Zhejiang University College of Medicine Hangzhou, China, Tel: +86-57188208188; E-mail: drwangwei@zju.edu.cn

Received: June 02, 2017; Accepted: June 02, 2017; Published: June 09, 2017

Citation: Wang W, Wang J (2017) Measurement of Nightmare Experience, and its Application in Research and Treatment of Psychiatric Disorders. J Psychiatry 20: e111. doi:10.4172/2378-5756.1000e111

Copyright: $\odot 2017$ Wang W, et al. This is an open-access article distributed under the terms of the Creative Commons Attribution License, which permits unrestricted use, distribution, and reproduction in any medium, provided the original author and source are credited 